\title{
Optical logic gate by the modulation of self-locking of a single mode FP-LD
}

\author{
M. Rakib Uddin ${ }^{1}$, J. S. Lim ${ }^{1}$, Y. D. Jeong ${ }^{2}$, and Y. H. Won ${ }^{1}$ \\ 1: Korea Advanced Institute of Science and Technology (KAIST), IT Convergence Campus, Munji-ro, Daejeon, South \\ Korea, e-mail: mmrakib@kaist.ac.kr.
}

2: Samsung Electronics, Suwon, South Korea

\begin{abstract}
An all-optical logic gate with NOR and NOT functions is proposed using a tunable single mode Fabry-Perot laser diode (FP-LD). A high on-off contrast ratio of over $40 \mathrm{~dB}$ is measured.
\end{abstract}

Keywords: All-optical logic gate, injection locking, single mode Fabry-Perot laser diode, optical signal processing.

\section{Introduction}

All-optical signal processing is strongly believed to be the most feasible technology that can provide the way out of the extreme limitations imposed on the capacity, speed and complexity of the present day communication and computation by conventional electronics. Optical devices and circuits have been incorporated into various systems and proved to be reliable and more advantageous. Optics provides higher bandwidth than electronics, which enables more information to be carried and data to be processed. Like other optical devices and circuits, all-optical logic gates have also received a considerable attention in the field of all-optical signal processing. To achieve all-optical logic functions, many approaches have been proposed on the basis of nonlinear effects either in optical fibers or semiconductor materials. Compared with their optical fiber based counter parts, logic gates using semiconductor optical amplifiers (SOAs) seem to be promising because of their transparent logic operations. However, most of the SOA based optical logic gates employ interferrometric structures requiring several SOAs, which makes the system somewhat complicated [1], [2]. For the last few years, researchers have been paying attention to an FP-LD and its applications in all-optical signal processing to find out a simple and cost-effective solution. All-optical logic gates using a multi-mode FP-LD have been then implemented, where external probe light sources are required [3], [4]. In [3], optical logic NOT and NOR functions have been realized by a dual injection locking mechanism in which both the pump and the probe beams must be locked with an FP-LD. Due to the dual injection locking, it is difficult to achieve a high on-off contrast ratio and the polarization and wavelength detuning dependency of the probe signal makes the system somewhat complicated and the system cost may be affected by the external tunable probe light source. In [4], optical logic gates based on absorption modulation was proposed. In the absorption modulation, an external tunable probe light source is required as well as an expensive and polarization sensitive polarization beam splitter (PBS) is needed to seperate transverse electric (TE) and transverse magnetic (TM) polarized lights in the system, which makes the system complex, expensive and impractical in real applications. In this paper, we propose a quite simple and low power consumed all-optical logic gates with a high on-off contrast ratio using the modulation of self-locking of a tunable single mode FP-LD. In the proposed scheme, there is no need for an external probe light source because the single mode FP-LD itself works well as a probe source. All-optical NOR and NOT functions are implemented to show an on-off contrast ratio of over $40 \mathrm{~dB}$ and the module can be operated with input power of less than $-5 \mathrm{dBm}$.

\section{Operation principle}

The operation principle of logic gates using a single mode FP-LD is based on the modulation of self-locking of a single mode FP-LD. The FP-LD we are using in this experiment is a new invention in our laboratory [5], specially designed with a built-in external cavity and operates in a single longitudinal mode with a high side mode suppression ratio. This special FP-LD is called a tunable single mode FP-LD or a self-locked FP-LD.

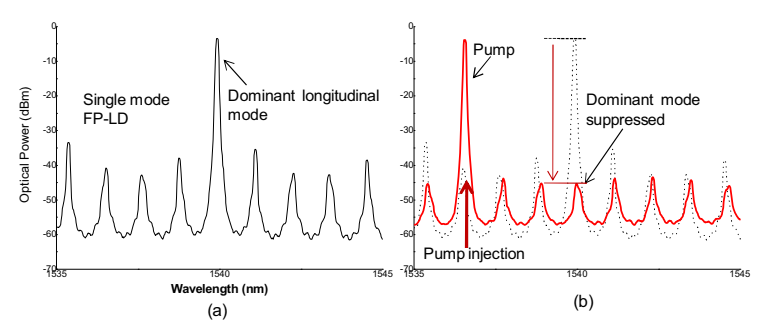

Fig. 1 : Operation principle. (a) Normal lasing of a single mode FP-LD. (b) After injection of a pump beam.

Fig. 1 explains the operation principle of a logic function using a single mode FP-LD. In Fig. 1 (a) the lasing view of the single mode FP-LD is represented without any external light injection. It has only one dominant longitudinal mode and all other side modes are suppressed. When any pump beam with TE polarized light is injected near to a longer wavelength side of any mode (dominant or low intensity mode) of the single mode FP-LD, the single mode operation of the FP-LD at the dominant longitudinal mode is collapsed to a very low level and the FP-LD locks to the injected beam as in Fig. 1 (b). The longitudinal mode of the FP-LD is collapsed because the injection locking on the selected mode by the pump beam causes the change of the carrier density that results in the red shift of laser cavity modes, and thus the mode matching condition of the coupled cavities is disturbed. If the input pump has sufficient power, the dominant longitudinal mode of the single mode FP-LD 
decreases to a very low level and in absence of input power the single longitudinal mode is high. Without any external pump injection, the level of the dominant longitudinal mode of a single mode FP-LD is high (logic ' 1 ') as in Fig. 1 (a) and with pump injection, the dominant mode is suppressed to a very low level (logic ' 0 ') as in Fig. 1 (b). By using this modulation effect of the dominant mode of a single mode FP-LD, we realize all-optical NOR and NOT gates in this experiment. By filtering out the dominant longitudinal mode wavelength of a single mode FP-LD, the logic output can be received.

\section{Experiment and results}

Fig. 2 shows a logic module for the experiment. The input signals for a logic operation were generated by tunable light sources modulated by $10 \mathrm{~Gb} / \mathrm{s}$ pseudorandom bit sequences. The wavelength of TL1 for input_A and TL2 for iput_B was set to $1536.56 \mathrm{~nm}$ and $1543.40 \mathrm{~nm}$, respectively with a detuning of $0.04 \mathrm{~nm}$ each. The FP-LD used in this experiment was biased at $18 \mathrm{~mA}$ and the operating temperature was $21^{\circ} \mathrm{C}$. The dominant longitudinal mode wavelength of single mode FP-LD was $1539.92 \mathrm{~nm}$. The power of the input signals before the gate operation was -5 $\mathrm{dBm}$ each.

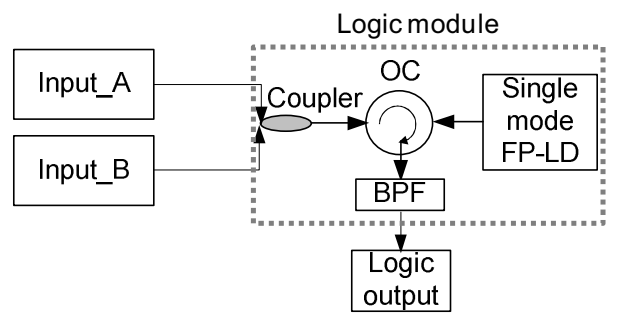

Fig. 2: Logic module. OC: optical circulator, BPF : optical bandpass filter.

The experimental results of NOR and NOT gates are shown in Fig. 3. It is mentioned that a single input NOR gate is a NOT gate and this NOT gate is the buffer circuit in optical domain. The significant observation is the high on-off contrast ratio and low power consumption of this scheme. Only $-5 \mathrm{dBm}$ input power for each pump is enough to operate the logic function. The on-off contrast ratio measures over $40 \mathrm{~dB}$ as shown in Fig. 3 (b), which can not be easily achievable in any other logic scheme reported untill now. The obtained high on-off contrast ratio results from the fact that when fully injection locking occurs in a single mode FP-LD by the injection of a TE polarized pump beam, the dominant longitudinal mode of a single mode FP-LD is fully suppressed. In dual injection locking logic gates [3], it is hard to obtain a high on-off contrast ratio because one external light (pump) cannot suppress another external light (probe) fully. The high on-off contrast ratio is very important for driving other devices connected to the output of the logic module. The oscilloscope traces of input-output waveforms of $10 \mathrm{~Gb} / \mathrm{s}$ data signals are shown in Fig. 3. By definition, the output in NOR gate is logic ' 1 ' if both the inputs are logic ' 0 ', otherwise output is logic ' 0 '. The results in Fig. 3 (c)-(e) support the truth table of NOR gate operation. For a single input it is a NOT gate and the results are shown in Fig. 3 (f)-(g).

\section{Conclusion}

All-optical logic functions of NOR and NOT gates using a single mode FP-LD is realized and a successful operation at $10 \mathrm{~Gb} / \mathrm{s}$ is reported. It doesn't require any external probe light source as required in other schemes. As a result, the proposed logic module is quite simple and attractive. The module needs very low input power for logic operations. Only $-5 \mathrm{dBm}$ input power for each pump is enough to operate the logic function. A high on-off contrast ratio of over $40 \mathrm{~dB}$ is also measured.
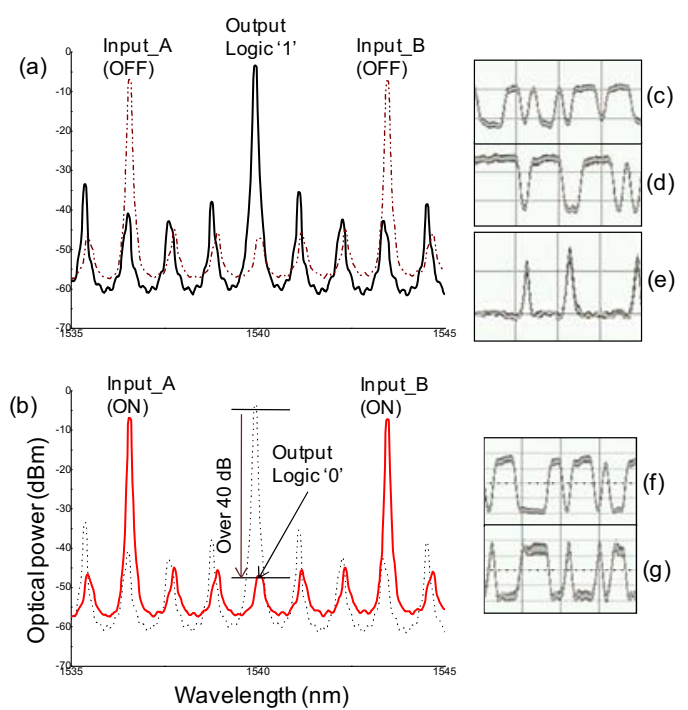

Fig. 3 : Experimental results. (a) Power spectra of NOR gate with both input 'OFF' state. (b) Power spectra of NOR gate with both input 'ON' state. (c), (d) waveforms of NOR gate inputs. (e) waveform of NOR gate output. (f) waveform of NOT gate input. (g) waveform of NOT gate output.

\section{References}

[1] R. P. Webb, R. J. Manning, G. D. Maxwell, and A. J. Poustie, "40 Gbit/s alloptical XOR gate based on hybrid-integrated Mach-Zehnder interferometer", Electronics Letters, vol. 39, no. 1 pp. 79-81, Jan. 2003.

[2] K. E. Stubkjaer, "Semiconductor optical amplifier-based all optical gates for high-speed optical processing", IEEE Journal on selected topics in quantum electronics, vol. 6, no. 6, pp. 1428-1435, 2000.

[3] L. Y. Chan, K. K. Qureshi, P. k. A. Wai, B. Moses, L. F. K. Lui, H. Y. Tam, M. S. Demokan, "All-optical bit-error monitoring system using cascaded inverted wavelength converter and optical NOR gate", IEEE photonic technology letters, vol. 15, no. 4, pp. 593-595, Apr. 2003.

[4] H. Yoo, H. J. Lee, Y. D. Jeong and Y. H. Won, "All-optical logic gates using absorption modulation of an injection-locked Fabry-Perot laser diode", International Conference on Photonics in Switching, Greece, Oct. 16-18, 2006.

[5] Y. D. Jeong, Y. H. Won, S. O. Choi, and J. H. Yoon, "Tunable single-mode Fabry-Perot laser diode using a built-in external cavity and its modulation characteristics", Optics Letters, vol. 31, no. 17, pp. 2586-2587, Sept. 2006. 